\title{
Microstrip Line on an Artificial Dielectric Substrate
}

\author{
Jan Machac, Senior Member, IEEE
}

\begin{abstract}
This letter presents a new version of an artificial dielectric substrate and a microstrip line built on top of this substrate. The substrate is fabricated as a two-dimensional array of metallic posts separated from the top conductor by a thin dielectric slab. This structure can show a very high effective permittivity and can therefore offer a substantial reduction in the dimensions of microwave circuit elements.
\end{abstract}

Index Terms-Artificial dielectric, dispersion characteristic, microstrip line, parallel plate waveguide, periodic structure.

\section{INTRODUCTION}

A RTIFICIAL dielectric materials have been used for more than 50 years [1]. These materials are a large-scale model of actual dielectric materials. A large number of conducting particles are arranged in a regular array. Under the action of an externally applied electric field, the charges on each conducting particle are displaced and simulate the behavior of the molecules in an ordinary dielectric. The original application of artificial dielectrics was to replace the heavy natural dielectric material in lens antennas [1]. More recently, various versions of artificial dielectrics have been used to modify the properties of materials in ways that are not attainable in nature, e.g., increasing the permittivity by a system of conducting strips [2]. These materials can also be used to reduce the permittivity [3], even below one [4], in order to improve the radiation properties of a leaky wave antenna by a rodded artificial dielectric [4]. Artificial dielectrics are widely used for forming various artificial surfaces, e.g., high impedance surfaces [5], and antireflection coatings [6]. The surface reactance can be effectively changed by using a system of short conducting pins [7].

This letter presents an artificial dielectric material suitable for building microstrip line based circuits. The material is formed by a two-dimensional (2-D) array of grounded metallic posts. A similar structure of a 2-D array of grounded cylindrical pins was used in [7], but with the aim to produce a surface with a given reactance. The posts are covered on their top by a very thin dielectric slab, on which the layout of the designed circuit is deposited. The substrate itself was tested in a parallel plate waveguide. Its effective permittivity was calculated by the CST Microwave Studio (MWS), and using the model of a line periodically loaded by capacitances. Effective permittivity up to several hundreds was predicted. This artificial substrate serves well as the substrate of a microstrip line that shows high effec-

Manuscript received January 11, 2006; revised April 3, 2006. This work was supported by the Czech Ministry of Education, Youth and Sports in the framework of the project "Research in the Area of the Prospective Information and Navigation Technologies" under Contract MSM 6840770014.

The author is with the Czech Technical University, Prague 16627, Czech Republic (e-mail: machac@fel.cvut.cz).

Digital Object Identifier 10.1109/LMWC.2006.877120 tive permittivity. It can thus substitute a microstrip line on an expensive high permittivity substrate, and can substantially reduce the dimensions of the circuit.

\section{Model of the Artificial Dielectric Material}

An artificial dielectric material can be represented by a threedimensional (3-D) array of dipoles terminated by impedance $Z_{L}$. The periods of the array $d x, d y$, and $d z$ must be considerably shorter than the wavelength, in order to be able to treat the medium as continuous. The effective length of the dipole arms is $l_{\text {eff. }}$ The incident electric field is oriented parallel to the dipole arms. The polarization vector is defined $P=p_{d} / V=\alpha_{e} E$, where $V=d x d y d z$ is the cell volume, $\alpha_{e}$ is the electric susceptibility that defines the permittivity of the medium, and $p_{d}$ is the dipole electric moment. The dipole input impedance has a capacitive character with capacitance $C_{e}$. Loading the dipole by capacitor $C_{L}$ we get the effective relative permittivity of this composite bulk material [8]

$$
\varepsilon_{\text {reff }}=1+\frac{\alpha_{e}}{\varepsilon_{0}}=1+\frac{1}{V \varepsilon_{0}} \frac{l_{\text {eff }}^{2} C_{e} C_{L}}{C_{e}+C_{L}} .
$$

Consequently, the medium created by the system of these dipoles shows artificially increased effective permittivity that according to (1) does not depend on frequency, supposing that $l_{\text {eff }}, C_{e}$ and $C_{L}$ do not depend on frequency.

Let us first study a parallel plate waveguide with the substrate made from this artificial dielectric material. The dipoles are represented by cuboidal metallic posts of dimensions $y_{p}, z_{p}$ and $h$ separated from the top conductor by a thin dielectric layer $h_{2}$ in thickness with permittivity $\varepsilon_{r}$ [see Fig. 1(a)]. The top and bottom conductors are represented by perfect electric (PEC) planes. Due to the periodicity of the field distribution, this infinitely wide and long structure can be analyzed as one row of posts, confined from the sides by perfect magnetic (PM) planes [Fig. 1(a)].

The line from Fig. 1(a) can be treated as a line periodically loaded by capacitors [9]. The dispersion equation of a bound wave on this line is [9]

$$
\cos (k p)=\cos \left(k_{0} d\right)-\frac{\bar{B}}{2} \sin \left(k_{0} d\right)
$$

where $k_{0}=\omega \sqrt{\mu_{0} \varepsilon_{0}}$ is the propagation constant in a free space, $k$ is the propagation constant of a wave on the line, $p$ is the line period, $d$ is the space between the posts, Fig. 1(a). $\bar{B}$ is the normalized susceptance of the post. This value can be roughly determined from $S_{11}$ of the single post calculated, e.g., by CST MWS

$$
\bar{B}=j \frac{2 S_{11}}{S_{11}+1} .
$$




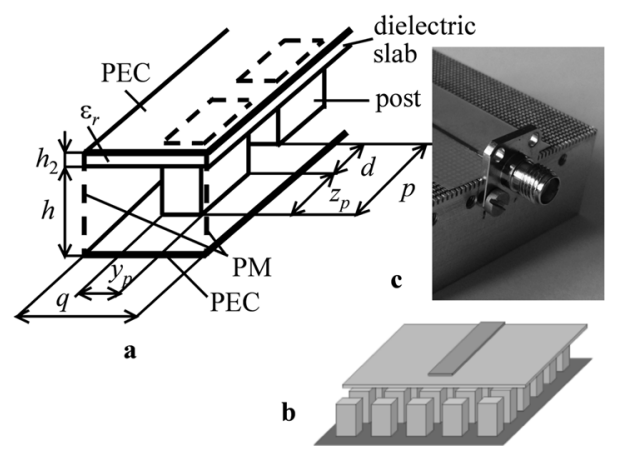

Fig. 1. (a) Ideal parallel plate waveguide with the artificial dielectric substrate, (b) the microstrip line on the artificial dielectric substrate, and (c) the fabricated line with the detail of the SMA connector.

The effective permittivity of the parallel plate waveguide is defined using propagation constant $k$ determined from (2)

$$
\varepsilon_{\text {eff }}=\left(k / k_{0}\right)^{2} .
$$

The low frequency limit of (2) gives the effective permittivity of the line defined by (4) in the form

$$
\varepsilon_{\text {eff }}=\left(\frac{d}{p}\right)^{2}\left(1+\frac{C_{p}}{C_{0} d}\right)
$$

where $C_{0}$ is the capacitance per unit length of the line without the posts $q$ in width

$$
C_{0}=\varepsilon_{0} q \frac{\varepsilon_{r}}{\varepsilon_{r} h+h_{2}} .
$$

$C_{p}$ is the capacitance of the post, which can be approximated assuming a very thin dielectric slab by

$$
C_{p}=\varepsilon_{0} \varepsilon_{r} \frac{y_{p} z_{p}}{h_{2}} .
$$

The problem in applying (4) is that in our line the posts are located rather close to each other and the susceptance (3) calculated for a single post does not give precise values. As will be shown below, this simple theory gives acceptable results for a very thin dielectric slab, i.e., for $h_{2} \rightarrow 0$.

The artificial dielectric material was produced by milling from a block of aluminium which at the same time represents the grounding plane [Fig. 1(c)]. The dimensions [see Fig. 1(a)] are $y_{p}=z_{p}=0.6 \mathrm{~mm}, d=0.4 \mathrm{~mm}, h=1 \mathrm{~mm}, q=1 \mathrm{~mm}$. The dielectric slab is a plastic foil with one-sided metallization. It is $h_{2}=0.09 \mathrm{~mm}$ in thickness, its permittivity is 2.9 , and the copper cladding is $0.035 \mathrm{~mm}$ in thickness. The effective permittivity of the line from Fig. 1(a) is plotted in Fig. 2. It was calculated directly by CST MWS and by (4). The experimental values were determined from the wavelength of the standing wave, the distribution of which was measured along the edge of the parallel plate waveguide with the top conductor $40 \mathrm{~mm}$ in width. The estimation of the effective permittivity by (4) is not precise in this case. Fig. 3 shows the effective permittivity

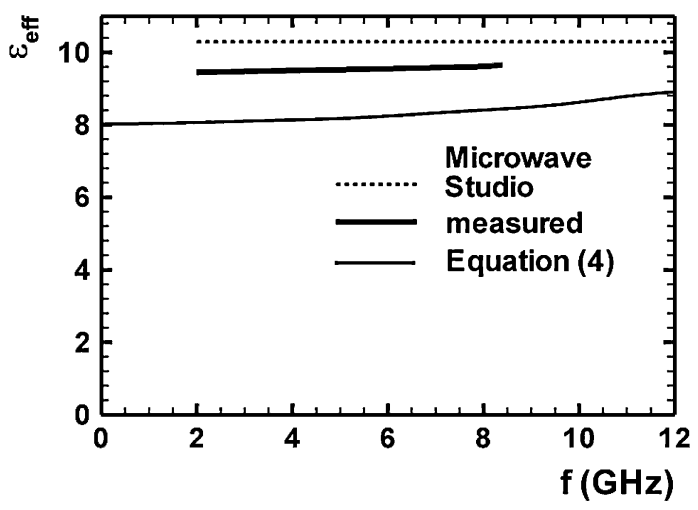

Fig. 2. Effective permittivity of the line from Fig. 1(a).

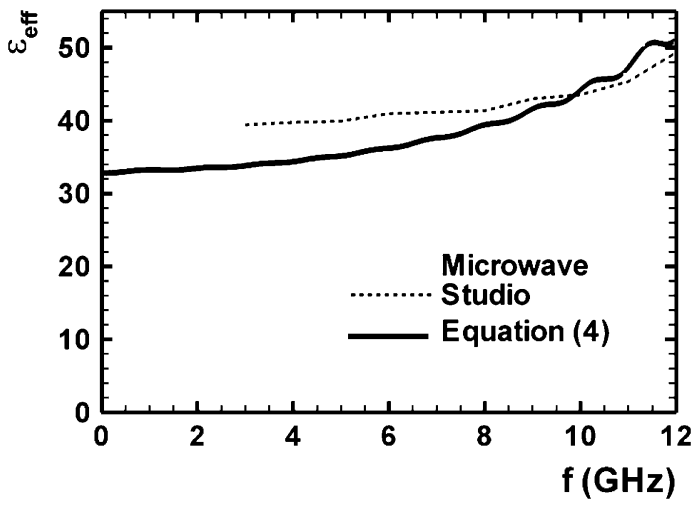

Fig. 3. Effective permittivity of the line from Fig. 1(a) with $h_{2}=10 \mu \mathrm{m}$ and $\varepsilon_{r}=2$, other parameters are stated in the text.

of the line from Fig. 1(a), where the dielectric layer is $10 \mu \mathrm{m}$ in thickness and permittivity $\varepsilon_{r}=2$. Equation (4) now offers a better estimate of the effective permittivity. The effective permittivity increases with decreasing thickness of the dielectric layer, due to the increase in $C_{p}$. For $h_{2}=1 \mu \mathrm{m}$ and $\varepsilon_{r}=$ 2 we get, using (4) at low frequencies, $\varepsilon_{\text {eff }}=288$, now well determined by (5)-(7). In contrast to the ideal case of a volume material (1), the effective permittivity of the line depends on the frequency, as the behavior of the post is frequency-dependent.

\section{Microstrip Line on AN ARTIFICIAL DIELECTRIC SUBSTRATE}

The proposed substrate was applied for a microstrip line. The CST MWS model of this line is shown in Fig. 1(b). The microstip line was designed and fabricated using the substrate defined above [see Fig. 1(c)]. The dielectric slab is $h_{2}=0.09 \mathrm{~mm}$ in thickness, $25 \mathrm{~mm}$ totally in width, and its permittivity is 2.9 . The microstrip line was fed via the SMA connectors as shown in Fig. 1(c). The phase constant of the bound wave propagating along this line with a strip $1.2 \mathrm{~mm}$ in width was calculated by CST MWS and determined from the wavelength of the standing wave, the distribution of which was measured along the line. Fig. 4 shows this phase constant recalculated to the line effective permittivity. The measured data fits the calculated effective permittivity of the line well.

The characteristic impedance of the microstrip line calculated by the CST Microwave Studio for various strip widths $w$ 


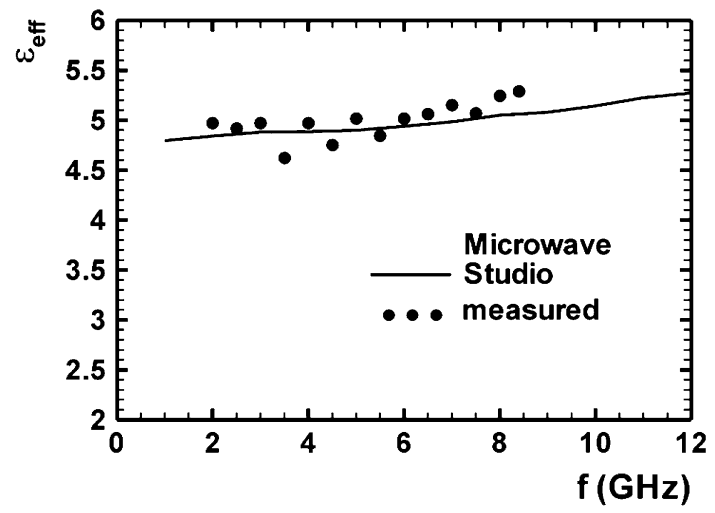

Fig. 4. Effective permittivity of the microstrip line on the artificial dielectric substrate from Fig. 1(b) with strip $1.2 \mathrm{~mm}$ in width.

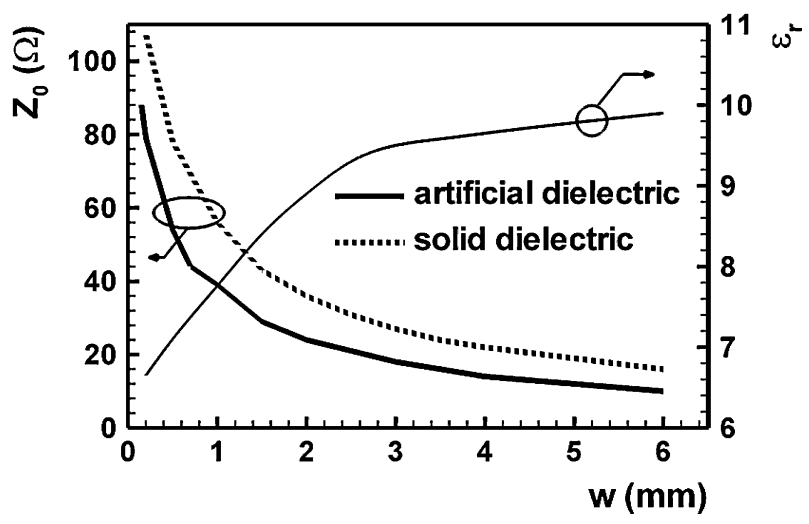

Fig. 5 Characteristic impedance of the microstrip line from Fig. 1(b) calculated by CST MWS at frequency $3 \mathrm{GHz}$. The relative permittivity of the solid dielectric substrate and the characteristic impedance of the microstrip line on this substrate (see the text).

is shown in Fig. 5. In Fig. 5, this impedance is compared with the calculated impedance of the microstrip line on a homogeneous solid dielectric substrate $h+h_{2}=1.09 \mathrm{~mm}$ in thickness, the permittivity of which $\varepsilon_{r}$ (see Fig. 5) was designed to get the microstrip line of the same effective permittivity as the line on the artificial substrate.

\section{CONCLUSION}

An artificial dielectric substrate is proposed in this letter. This substrate consists of a 2-D array of grounded cuboidal metallic posts on top of which a thin dielectric slab is placed. This substrate is a high permittivity medium. It was tested as the substrate of a parallel plate waveguide. The effective permittivity of this waveguide can reach values up to several hundreds, depending on the dimensions of the posts, the thickness, and the permittivity of the dielectric slab. This substrate is suitable for microstrip line based circuits. The microstrip line on an artificial substrate behaves similarly as a standard microstrip line on a homogeneous dielectric substrate. A microstrip line on an artificial dielectric substrate was fabricated. The measured effective permittivity corresponds well to the data calculated by CST MWS. The characteristic impedance of this line was calculated.

The proposed artificial dielectric substrate itself, and the microstrip line designed on it, are suitable building blocks for microwave circuits. Due to the high effective permittivity, they can substantially reduce the dimensions of circuits such as resonators, filters, and couplers.

\section{REFERENCES}

[1] W. E. Kock, "Metallic delay lenses," Bell Syst. Tech. J., vol. 27, pp. $58-82,1948$

[2] I. Awai, H. Kubo, T. Iribe, D. Wakamiya, and A. Sanada, "An artificial dielectric material of huge permittivity with novel anisotropy and its application to a microwave BPF," in IEEE MTT-S Dig., Philadelphia, PA, Jun. 2003, vol. 1, pp. 301-304.

[3] K. F. Sabet, T. Ozdemir, P. Frantzis, K. Sarabandi, and L. P. B. Katehi, "Compact wireless antennas using an artificial dielectric lens," in Proc. IEEE Aerosp. Conf., 2002, vol. 2, pp. 931-937.

[4] I. J. Bahl and P. Bhartia, "Leaky-wave antennas using artificial dielectrics at millimeter wave frequencies," IEEE Trans. Microw. Theory Tech., vol. MTT-28, no. 11, pp. 1205-1212, Nov. 1980.

[5] R. E. Diaz, J. T. Aberle, and W. E. McKinzie, "TM mode analysis of a Sievenpiper high-impedance reactive surface," in IEEE Antennas Propag. Soc. Int. Symp. Dig., Jun. 2000, pp. 327-330.

[6] S. Biber, J. Richter, S. Martius, and L.-P. Schmidt, "Design of artificial dielectrics for ant-reflection-coatings," in Proc. 33rd Eur. Microw. Conf., Munich, Germany, Oct. 2003, pp. 1115-1118.

[7] R. J. King, D. V. Thiel, and K. S. Park, "The synthesis of surface reactance using an artificial dielectric," IEEE Trans. Antennas Propag., vol. AP-31, no. 3, pp. 471-476, May 1983.

[8] R. W. Ziolkowski and F. Auzanneau, "Passive artificial molecule realizations of dielectric materials," J. Appl. Phys., vol. 82, no. 7, pp. 3195-3198, Oct. 1997.

[9] R. E. Collin, Foundations for Microwave Engineering, 2nd ed. Pistacaway, NJ: IEEE Press, 2001. 